\title{
Stabilized Reduced Basis Approximation of Incompressible Three-Dimensional Navier-Stokes Equations in Parametrized Deformed Domains
}

\author{
Simone Deparis • A. Emil Løvgren
}

Received: 26 April 2010 / Revised: 11 February 2011 / Accepted: 28 February 2011 /

Published online: 17 March 2011

(C) Springer Science+Business Media, LLC 2011

\begin{abstract}
In this work we are interested in the numerical solution of the steady incompressible Navier-Stokes equations for fluid flow in pipes with varying curvatures and crosssections. We intend to compute a reduced basis approximation of the solution, employing the geometry as a parameter in the reduced basis method. This has previously been done in a spectral element $P_{\mathcal{N}}-P_{\mathcal{N}-2}$ setting in two dimensions for the steady Stokes equations. To compute the necessary basis-functions in the reduced basis method, we propose to use a stabilized $P_{1}-P_{1}$ finite element method for solving the Navier-Stokes equations on different geometries. By employing the same stabilization in the reduced basis approximation, we avoid having to enrich the velocity basis in order to satisfy the inf-sup condition. This reduces the complexity of the reduced basis method for the Navier-Stokes problem, while keeping its good approximation properties. We prove the well posedness of the reduced problem and present numerical results for selected parameter dependent three dimensional pipes.
\end{abstract}

Keywords Reduced basis methods · Steady incompressible Navier-Stokes equations · Stabilization

\section{Introduction}

The approximation of the Navier-Stokes equations in deformable domains, like in fluidstructure interaction, in shape optimization and control [16], is often expensive or requires model order reduction to speed up the computations. One way to achieve this reduction is by the reduced basis method, that applies to parameter dependent partial differential equations.

S. Deparis $(\bowtie)$

CMCS, MATHICSE, EPFL, Station 8, 1015 Lausanne, Switzerland

e-mail: simone.deparis@epfl.ch

A.E. Løvgren

SIMULA Research Laboratory, P.O. Box 134, 1325 Lysaker, Norway

e-mail: emil@simula.no 
Under the assumption that the solution resides on a smooth manifold with respect to the parameter, the solution of the given problem for a specific choice of the parameter is found as a linear combination of precomputed solutions, or basis-functions, through a Galerkin method [13, 14].

The geometry of the domain is parametrized through a change of variables and the equations are expressed on the reference domain; this involves the Jacobian of the map from the reference domain to the deformed parameter-dependent domain (e.g. [11]). By changing the deformed domain, the Jacobian in the equations change accordingly.

The idea of the reduced basis method is to separate the problem into an offline stage and an online stage. In the offline stage, $N$ basis-functions are computed by solving the parameter dependent problem for different choices of the parameter. The basis-functions are computed on a finite element grid which is fine enough to sufficiently resolve the problem. Typically, $N$ is $\mathcal{O}(10)$, or $\mathcal{O}(100)$, and $N \ll \mathcal{N}$, where $\mathcal{N}$ is the number of degrees-offreedom in the finite element approximation. In the online stage, the reduced basis approximation for a new value of the parameter is found by identifying $N$ coefficients, in a fraction of the time it takes to compute the finite element solution. When the output of interest is a derived quantity, e.g. a functional of the solution instead of the solution itself, and the problem has affine parameter dependence, the output of interest together with effective error bounds can be computed with online complexity independent of the finite element degreesof-freedom [15].

The reduced basis element method is a variation of the established reduced basis method where the geometry of the computational domain is used as a parameter and the computational domain is decomposed into several building blocks [8,9]. Domain decomposition is used to identify a generic geometry as a union of building blocks, where each building block is found as a one-to-one map of one of a limited number of reference domains. Adhering to each reference domain is a set of precomputed basis-functions, which are computed in the offline stage by solving the underlying equations on several different maps of the reference domain, and then mapped back to the reference domain and stored in the reference coordinate system. Scalar basis-functions are mapped through composition with the reference variables, while vector basis-functions in addition are rotated and scaled by the Piola transformation which guarantees the incompressibility and preserves the local orientation of the vector-fields with respect to the boundary of the domain [2].

In the online stage, the reduced basis element approximation on each subdomain of the generic geometry is found through a Galerkin projection on a space generated by the precomputed basis-functions. The basis-functions are now mapped from the proper reference domain to each generic subdomain. Across subdomain interfaces, low order polynomials are used as Lagrange multipliers to ensure weak continuity. The reduced basis element method was developed by Maday and Rønquist in [11, 12] for heat conduction in two-dimensional affine mappings, and by Løvgren et al. in $[8,9]$ for steady Stokes flow, also in two dimensions, but for non-affine mappings. In [16], Rozza used several affine geometric parameters in the reduced basis method for optimizing the shape of a two-dimensional by-pass configuration for a blood vessel. In a recent article, Chen et al. [4] propose the use of stabilized discontinuous Galerkin approximation in the context of reduced basis element method for the time-harmonic Maxwell's equation in order to simplify the glueing of the macro elements.

This work considers only one reference domain and one generic subdomain. Although it is a key ingredient for an efficient offline/online decomposition and also for the construction of an optimal reduced basis, we neglect here any form of a posteriori error estimation.

There are two new aspects to the reduced basis method in this work. The first is the extension of the flow model to the Navier-Stokes equations in three dimensions. We thus have 
a non-linear model for the flow, and in addition a much richer space for geometric deformations, e.g. twisting and bending along several axes. We restrict the model to stationary flow and deformation only in a plane, but the methodology is quite general.

The second new aspect in this work is the use of the reduced basis method together with the stabilized $P_{1}-P_{1}$ finite element approximation presented by Burman et al. in [3]. The use of equal order elements for pressure and velocity is practical from a computational point of view, and the inf-sup condition is fulfilled by introducing stabilization terms in the weak formulation of the finite element problem. We use the same stabilization terms in the reduced basis problem, and prove that the reduced basis problem is well posed. Moreover the computation of the reduced basis velocity and pressure solution can be split in two sequential sub-problems, without loosing precision; indeed with a higher precision and a lower condition number of the linear system.

As an ingredient of the three-dimensional modelling we introduce the reduced basis element framework in an existing finite element library, LifeV [7]. The intention is to reuse the existing operators in the library, and insert the necessary reduced basis operators in a layer on top of the existing solvers.

In Sect. 2.1 we present the weak form of the Navier-Stokes equations, followed by the finite element formulation in Sect. 3.1 and the reduced basis formulation in Sect. 3.2, where we also prove the well posedness of the reduced problem. In Sect. 3.3 we include some details of the construction of the reduced basis ingredients. Section 4 is devoted to numerical results. Finally, we give some concluding remarks in Sect. 5.

\section{Problem Statement}

Our model problem is the simulation of pressure driven flow through pipes, where the shape of the pipes changes from case to case. Applications include blood-flow through vessels where the geometry is found from patient specific data obtained from MR imaging, and air flowing in the respiratory system. Also engineering problems where the geometry and layout of pipes are considered in, e.g., cooling and heating systems could be of interest. Bifurcating geometries can be included for more complex flow systems.

The flow is modeled by the incompressible Navier-Stokes equations, where the primary unknowns are the pressure, $p$, and the velocity, $\boldsymbol{u}$, in the rigid domain $\Omega$. One end of the domain is defined as an inlet boundary, $\Gamma^{\mathrm{In}}$, and the other end as an outlet boundary, $\Gamma^{\text {Out }}$. We impose no slip boundary conditions on the sides of the domain $\Gamma^{D}$. On this domain we seek $(\boldsymbol{u}, p)$ such that

$$
\begin{aligned}
-v \Delta \boldsymbol{u}+(\boldsymbol{u} \cdot \nabla) \boldsymbol{u}+\nabla p=0 & \text { in } \Omega, \\
\nabla \cdot \boldsymbol{u}=0 & \text { in } \Omega,
\end{aligned}
$$

with homogeneous Dirichlet boundary conditions, $\boldsymbol{u}=0$, on $\Gamma^{D}$, natural boundary conditions, $\boldsymbol{\sigma} \cdot \boldsymbol{n} \equiv-p \boldsymbol{n}+v \partial_{\boldsymbol{n}} \boldsymbol{u}=1$ on $\Gamma^{\mathrm{In}}$, and homogeneous natural boundary conditions on $\Gamma^{\text {Out }}$, i.e, $\boldsymbol{\sigma} \cdot \boldsymbol{n}=0$. Here $\boldsymbol{\sigma}$ is the stress tensor related to the fluid, and $\boldsymbol{n}$ is the outward facing normal on the given boundary.

When discretizing the Stokes or Navier-Stokes equations by a Galerkin projection, the finite element spaces for the pressure and velocity have to be inf-sup stable, i.e.,

$$
\inf _{q \in M} \sup _{\boldsymbol{v} \in X} \frac{\int_{\Omega} q \nabla \cdot \boldsymbol{v} d \Omega}{\|q\|_{L^{2}(\Omega)}\|\boldsymbol{v}\|_{H^{1}(\Omega)}} \geq \beta>0,
$$


where $M$ is the pressure space, $X$ is the velocity space, and $\beta$ is a positive constant. If the infsup condition is not satisfied the problem is not well-posed. Typically, if the velocity finite element space consists of piecewise quadratic basis-functions, the corresponding pressure space consists of piecewise linear basis-functions. In the reduced basis method for the Stokes and Navier-Stokes problem, different methods of enriching the velocity basis are used to guarantee a lower bound for the inf-sup constant, $\beta$; see $[8,17]$.

In case the spaces for the pressure and velocity do not satisfy the inf-sup condition, Burman et al. [3, 19] introduced interior penalty stabilization methods in order to get a wellposed problem. This allows for expressing both the velocity and the pressure on the same finite element basis, here continuous piecewise linear elements $P_{1}$. Due to the stabilization term however, the velocity solutions are not exactly incompressible.

We use the stabilized $P_{1}-P_{1}$ method presented below to compute our basis-functions for the reduced basis method. In addition, we propose to use the same stabilization term directly in our reduced basis approximation. Therefore it is not necessary to enrich the velocity basis in order to satisfy the inf-sup condition for the reduced basis problem.

\subsection{Continuous Problem}

We consider a straight pipe as our reference domain, $\widehat{\Omega}$ and an actual geometry described by $P$ independent parameters $\mu \in \mathbb{R}^{P}$. We let $\Phi_{\mu}$ define a one-to-one map from the reference domain to a deformed domain $\Omega_{\mu}$ :

$$
\Phi_{\mu}: \widehat{\Omega} \rightarrow \Omega_{\mu} .
$$

In Fig. 1 we present the reference pipe to the left, and a deformed pipe found by bending the reference domain to the right. This is a simple deformation used for illustration, and the list of possible deformations is endless. What we assume in this work, is that all maps $\Phi_{\mu}$ are one-to-one and at least $C^{1}$.

On the deformed domain we define the bi-linear and tri-linear forms

$$
\begin{aligned}
a(\boldsymbol{u}, \boldsymbol{v} ; \boldsymbol{\mu}) & =v \int_{\Omega_{\mu}} \nabla \boldsymbol{u}: \nabla \boldsymbol{v} d \Omega_{\boldsymbol{\mu}}, \\
b(\boldsymbol{v}, q ; \boldsymbol{\mu}) & =-\int_{\Omega_{\mu}} q \nabla \cdot \boldsymbol{v} d \Omega_{\mu}, \\
c(\boldsymbol{u}, \boldsymbol{w}, \boldsymbol{v} ; \boldsymbol{\mu}) & =\int_{\Omega_{\mu}}(\boldsymbol{w} \cdot \nabla) \boldsymbol{u} \boldsymbol{v} d \Omega_{\mu},
\end{aligned}
$$

Fig. 1 On the left is the reference domain $\widehat{\Omega}$ and on the right a generic deformed domain $\Omega_{\mu}$

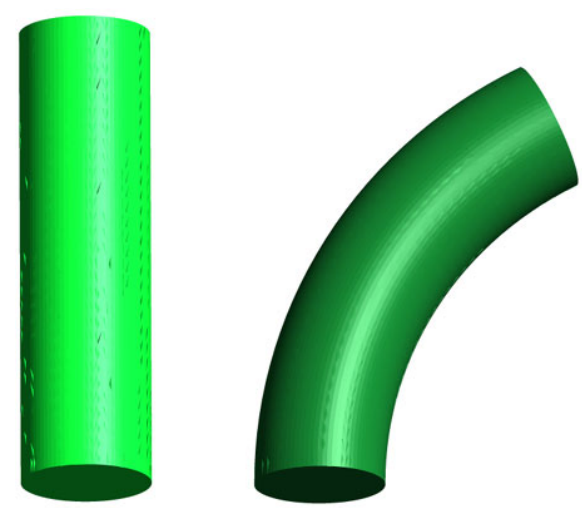


where $\boldsymbol{u}, \boldsymbol{v}$, and $\boldsymbol{w}$ are velocity fields, $q$ is a scalar pressure field, and $v$ is the viscosity. In this work we focus on the geometric deformation and set $v=1$.

Furthermore, we define the space $V\left(\Omega_{\mu}\right)=\left\{\boldsymbol{v} \in\left(H^{1}\left(\Omega_{\mu}\right)\right)^{3}:\left.\boldsymbol{v}\right|_{\Gamma^{D}}=0\right\} \times L^{2}\left(\Omega_{\mu}\right)$. The weak form of the steady incompressible Navier-Stokes equations on the deformed domain then reads: find $(\boldsymbol{u}, p)$ in $V\left(\Omega_{\mu}\right)$ such that

$$
\left.\begin{array}{rl}
a(\boldsymbol{u}, \boldsymbol{v} ; \boldsymbol{\mu})+c(\boldsymbol{u}, \boldsymbol{u}, \boldsymbol{v} ; \boldsymbol{\mu})+b(\boldsymbol{v}, p ; \boldsymbol{\mu}) & =l(\boldsymbol{v} ; \boldsymbol{\mu}) \\
b(\boldsymbol{u}, q ; \boldsymbol{\mu}) & =0
\end{array}\right\} \quad \forall(\boldsymbol{v}, q) \in V\left(\Omega_{\boldsymbol{\mu}}\right),
$$

where $l(\boldsymbol{v} ; \boldsymbol{\mu})=\int_{\Gamma_{\boldsymbol{\mu}}^{\mathrm{In}}} \boldsymbol{\sigma} \cdot \boldsymbol{n} \cdot \boldsymbol{v} d s$. The problem is non-linear, and in the next section we present one of the possible methods for dealing with this non-linearity.

\section{Discretization}

\subsection{Finite Element Formulation of the Oseen Problem}

We carry out the numerical analysis of our problem for the Oseen equations. These are characterized by a convective vector-field $\boldsymbol{w}$ that enters in (3) as $c(\cdot, \boldsymbol{w}, \cdot ; \boldsymbol{\mu})$. One way of solving the steady Navier-Stokes equations is to consider pseudo time-dependence, take as value of $\boldsymbol{w}$ the value of the velocity at the previous time-step, solve the Oseen equations, and repeat until a steady state is reached.

Let $\widehat{\mathcal{T}}_{h}$ be a regular tetrahedral mesh of the reference configuration. This mesh, i.e., the vertices of the tetrahedrons, is mapped to a deformed domain through the map $\Phi_{\mu}$. We suppose that the mapping is regular enough such that the resulting mesh $\mathcal{T}_{h}$ is still regular. In other words, the allowed values of the parameter $\boldsymbol{\mu}$ are such that the mapped mesh is regular (avoiding negative local mass or overlapping elements). We then define $P_{1}-P_{1}$ finite elements on the deformed domain $\Omega_{\mu}$ by

$$
V_{h}\left(\Omega_{\mu}\right)=\left\{(\boldsymbol{v}, p) \in\left(C^{0}\left(\Omega_{\mu}\right)\right)^{3} \times C^{0}\left(\Omega_{\boldsymbol{\mu}}\right):\left.\boldsymbol{v}\right|_{\Gamma^{D}}=0,\left.\boldsymbol{v}\right|_{K} \in P_{1} \text { and }\left.p\right|_{K} \in P_{1} \forall K \in \mathcal{T}_{h}\right\},
$$

where $P_{1}$ represents first order polynomials. In addition, we let $V_{h}^{u}\left(\Omega_{\mu}\right)$ denote the velocity components of $V_{h}\left(\Omega_{\mu}\right)$, and $V_{h}^{p}\left(\Omega_{\mu}\right)$ the pressure components.

We introduce a stabilization term in order to make the discretization inf-sup stable, similarly to $[3,19]$

$$
\begin{aligned}
& \hat{j}_{p}(\hat{p}, \hat{q})=\int_{\hat{\Gamma}_{I}} \gamma \frac{h_{f}^{3}}{v} \llbracket \nabla \hat{p} \rrbracket_{f} \cdot \llbracket \nabla \hat{q} \rrbracket_{f} d \hat{s}, \\
& j_{p}(p, q ; \boldsymbol{\mu})=\hat{j}_{p}\left(p \circ \Phi_{\mu}, q \circ \Phi_{\mu}\right),
\end{aligned}
$$

where $\gamma$ is a dimensionless positive constant, $\hat{\Gamma}_{I}$ is the union of all the internal faces of our reference mesh, $h_{f}$ is the diameter of a face, and $\llbracket \cdot \rrbracket_{f}$ is the jump of a quantity across a face (note that the gradients of $p$ and $q$ are not continuous across a face). With respect to [3, 19], we assume that $h_{f}|\boldsymbol{u}|<v$, where $|\boldsymbol{u}|$ is the modulus of the velocity. A further difference is that the stabilization contributions are done with respect to the reference domain; however the two formulations are equivalent up to a change in the stabilization parameters depending on the families $\left\{\widehat{\mathcal{T}}_{h}\right\}_{h}$ and $\left\{\mathcal{T}_{h}\right\}_{h}$. This is needed to guarantee good approximation properties of the reduced basis approximation and is used in the well-posedness proof. It is possible to also include other stabilization terms for the convection as presented in $[3,19]$. 
The finite element problem then reads: find $(\boldsymbol{u}, p)$ in $V_{h}\left(\Omega_{\mu}\right)$ such that

$$
\begin{aligned}
& a(\boldsymbol{u}, \boldsymbol{v} ; \boldsymbol{\mu})+c(\boldsymbol{u}, \boldsymbol{u}, \boldsymbol{v} ; \boldsymbol{\mu})+b(\boldsymbol{v}, p ; \boldsymbol{\mu})-b(\boldsymbol{u}, q ; \boldsymbol{\mu})+j_{p}(p, q ; \boldsymbol{\mu})=l(\boldsymbol{v} ; \boldsymbol{\mu}) \\
& \quad \forall(\boldsymbol{v}, q) \in V_{h}\left(\Omega_{\boldsymbol{\mu}}\right),
\end{aligned}
$$

Since the problem is non-linear, we solve it by pseudo time-advancing until the steady solution is reached. In this respect we need an artificial time-step $\Delta t$ and a bi-linear form

$$
m(\boldsymbol{u}, \boldsymbol{v} ; \boldsymbol{\mu})=\frac{1}{\Delta t} \int_{\Omega_{\mu}} \boldsymbol{u} \cdot \boldsymbol{v} d \Omega_{\mu} .
$$

Starting from an initial guess $\left(\boldsymbol{u}^{0}, p^{0}\right)$ we implement the following algorithm: given $\left(\boldsymbol{u}^{n}, p^{n}\right)$ in $V_{h}\left(\Omega_{\mu}\right)$, find $\left(\boldsymbol{u}^{n+1}, p^{n+1}\right)$ in $V_{h}\left(\Omega_{\mu}\right)$ such that

$$
\begin{aligned}
& m\left(\boldsymbol{u}^{n+1}, \boldsymbol{v} ; \boldsymbol{\mu}\right)+a\left(\boldsymbol{u}^{n+1}, \boldsymbol{v} ; \boldsymbol{\mu}\right)+c\left(\boldsymbol{u}^{n+1}, \boldsymbol{u}^{n}, \boldsymbol{v} ; \boldsymbol{\mu}\right)+b\left(\boldsymbol{v}, p^{n+1} ; \boldsymbol{\mu}\right) \\
& \quad-b\left(\boldsymbol{u}^{n+1}, q ; \boldsymbol{\mu}\right)+j_{p}\left(p^{n+1}, q ; \boldsymbol{\mu}\right)=l(\boldsymbol{v} ; \boldsymbol{\mu})+m\left(\boldsymbol{u}^{n}, \boldsymbol{v} ; \boldsymbol{\mu}\right) \quad \forall(\boldsymbol{v}, q) \in V_{h}\left(\Omega_{\boldsymbol{\mu}}\right) .
\end{aligned}
$$

At each time-step we solve this linear problem using a preconditioned iterative method (PGMRES). We choose $\left(\boldsymbol{u}^{n}, p^{n}\right)$ as an initial guess for the linear solver at the next timestep such that convergence of the linear solver in zero iterations means that we have reached the steady state solution (5).

\subsection{The Reduced Basis Method}

To construct the basis-functions for the reduced basis method, we define the parameter set $S_{N}$ by $N$ samples $\left\{\boldsymbol{\mu}^{(i)}\right\}_{i=1}^{N}$. Each sample $\boldsymbol{\mu}^{(i)} \in \mathcal{D} \subset \mathbb{R}^{P}$ defines a one-to-one map from the reference domain to a sample geometry, $\Phi^{(i)}=\Phi_{\mu^{(i)}}: \widehat{\Omega} \rightarrow \Omega_{\mu^{(i)}}$. On each sample geometry we find $\left(\boldsymbol{u}^{(i)}, p^{(i)}\right) \in V_{h}\left(\Omega_{\boldsymbol{\mu}^{(i)}}\right)$ such that

$$
\begin{aligned}
& a\left(\boldsymbol{u}^{(i)}, \boldsymbol{v} ; \boldsymbol{\mu}^{(i)}\right)+c\left(\boldsymbol{u}^{(i)}, \boldsymbol{u}^{(i)}, \boldsymbol{v} ; \boldsymbol{\mu}^{(i)}\right)+b\left(\boldsymbol{v}, p^{(i)} ; \boldsymbol{\mu}^{(i)}\right) \\
& \quad-b\left(\boldsymbol{u}^{(i)}, q ; \boldsymbol{\mu}^{(i)}\right)+j_{p}\left(p^{(i)}, q ; \boldsymbol{\mu}\right)=l\left(\boldsymbol{v} ; \boldsymbol{\mu}^{(i)}\right) \quad \forall(\boldsymbol{v}, q) \in V_{h}\left(\Omega_{\boldsymbol{\mu}^{(i)}}\right),
\end{aligned}
$$

by the pseudo time-advancing procedure described in Sect. 3.1. The basis-functions are then mapped to the reference domain and stored in the reference variables.

The pressure fields are scalar and are mapped as such through composition with the maps $\Phi_{\mu}^{(i)}$. The velocities are vector-fields and, in order to keep their orientation with respect to the inflow and outflow boundaries and to preserve the divergence of the vector-fields, we use the Piola transformation to map them between the different geometries. The Piola transformation, who's general properties are defined in [2], is defined as the transformation of the vector-field $v$ from $\Omega_{\mu}$ to $\widehat{\Omega}$ given by

$$
\hat{\boldsymbol{v}}=\Psi_{\mu}(\boldsymbol{v})=\left(\mathcal{J}_{\mu}\right)^{-1}\left(\boldsymbol{v} \circ \Phi_{v}\right)\left|J_{\mu}\right|
$$

Here $\mathcal{J}_{\mu}$ is the Jacobian matrix of the map $\Phi_{\mu}$ and $\left|J_{\mu}\right|$ is the absolute value of its determinant. Since the velocity solutions of (5), found using the stabilization method, are not completely incompressible, the Piola transformed velocities are not incompressible on the reference domain. The discretization of the Piola transformation also contributes to some loss in the divergence free properties of a vector-field; in general, for $q \in V_{h}^{p}\left(\Omega_{\mu}\right), \hat{q}=q \circ \Phi_{\mu}$ 
is in $V_{h}^{p}(\hat{\Omega})$, while for $\boldsymbol{v} \in V_{h}\left(\Omega_{\mu}\right)$, $\hat{\boldsymbol{v}}=\Psi_{\mu}(\boldsymbol{v})$ is not in $V_{h}^{\boldsymbol{u}}(\hat{\Omega})$. Our discretized problem takes into account this difficulty by computing some of the components in the reduced basis system matrix with respect to the reference finite element configuration.

If we assume that the Piola transformation is not approximated, we have that

$$
b(\boldsymbol{v}, q ; \boldsymbol{\mu})=-\int_{\Omega_{\mu}} q \nabla \cdot \boldsymbol{v} d \Omega_{\mu}=-\int_{\hat{\Omega}} \hat{q} \nabla \cdot \hat{\boldsymbol{v}} d \Omega=\hat{b}(\hat{\boldsymbol{v}}, \hat{q})
$$

for $\hat{\boldsymbol{v}}=\Psi_{\mu}(\boldsymbol{v})$ and $\hat{q}=q \circ \Phi_{\mu}$. In practice, this is not the case; moreover the vector-fields on the reference domain are projected on the space $V_{h}(\hat{\Omega})$. The following Lemma holds only if the Piola transformation is not approximated:

Lemma 1 For a given $\boldsymbol{\mu}$ in $\mathcal{D}$, let $(\boldsymbol{u}, p) \in V_{h}\left(\Omega_{\boldsymbol{\mu}}\right)$ be the solution of (5), with $\hat{\boldsymbol{u}}=\Psi_{\boldsymbol{\mu}}(\boldsymbol{u})$ and $\hat{p}=p \circ \Phi_{\mu}$. Let

$$
\hat{b}(\hat{\boldsymbol{v}}, \hat{q}) \equiv-\int_{\hat{\Omega}} \hat{q} \nabla \cdot \hat{\boldsymbol{v}} d \Omega
$$

then for all $q$ in $V_{h}^{p}(\Omega)$

$$
\hat{b}\left(\hat{\boldsymbol{u}}, q \circ \Phi_{\mu}\right)=\hat{j}_{p}\left(\hat{p}, q \circ \Phi_{\mu}\right) .
$$

Proof The Lemma is proved by Property (9) and (5).

On the reference domain the basis-functions are normalized, otherwise the condition number of the reduced system grows uncontrollably. There are several options when choosing the inner product in the modified Gram-Schmidt procedure. Usually for the approximation of the Stokes and Navier-Stokes equations by the reduced basis method the velocity basis-functions and the pressure basis-functions are orthonormalized independently $[5,16]$. Instead, before splitting the velocity and the pressure basis functions, we orthonormalize the vectors $\left\{\left(\hat{\boldsymbol{u}}^{(i)}, \hat{p}^{(i)}\right)\right\}_{i=1}^{N}$ with respect to the norm induced by $H^{1}(\hat{\Omega})^{3}$ and $\hat{j}(\cdot, \cdot)$. (Indeed, $\hat{j}$ induces a seminorm on $L^{2}(\hat{\Omega})$, thus the normalization procedure may fail; however we noticed that the condition number of the reduced-basis system is smaller with this choice instead of the $L^{2}$-norm.)

This allows us to prove the following property:

Lemma 2 Let $\left\{\left(\hat{\boldsymbol{u}}^{(i)}, \hat{p}^{(i)}\right)\right\}_{i=1}^{N}$ be the (already) orthonormalized basis-functions on the reference domain, then for all $\hat{q}$ in $V_{h}^{p}(\hat{\Omega})$

$$
\hat{b}\left(\hat{\boldsymbol{u}}^{(i)}, \hat{q}\right)=\hat{j}_{p}\left(\hat{p}^{(i)}, \hat{q}\right) .
$$

Proof The claim follows from the bi-linearity of $\hat{b}$ and $\hat{j}$, Lemma 1 , as well as from the interdependent orthonormalization procedure.

On a generic domain, $\Omega_{\mu}$, we define the reduced basis approximation space

$$
\begin{aligned}
V_{N}^{u}\left(\Omega_{\mu}\right) & =\mathcal{L}\left\{\Psi_{\mu}^{-1}\left(\hat{\boldsymbol{u}}^{(i)}\right), i=1, \ldots, N\right\}, \\
V_{N}^{p}\left(\Omega_{\mu}\right) & =\mathcal{L}\left\{\hat{p}^{(i)} \circ \Phi_{\mu}^{-1}, i=1, \ldots, N\right\} .
\end{aligned}
$$


We say that $\left\{p^{(i)}\right\}_{i=1}^{N}$ are $\hat{j}_{p}$-linear independent if the following property holds:

$$
\sum_{i=1}^{N} \alpha_{i} \hat{j}_{p}\left(\hat{p}^{(i)}, \hat{p}^{(j)}\right)=0 \quad \forall j=1, \ldots, N \quad \Longrightarrow \quad \alpha_{i}=0 \quad \forall i=1, \ldots, N .
$$

This means that $\left\{p^{(i)}\right\}_{i=1}^{N}$ are linear independent and that $\hat{j}$ defines a scalar product in the space generated by these vectors. This property has to be verified offline by a Gram-Schmidt that uses $\hat{j}$ as scalar product.

The reduced basis approximation of the steady Navier-Stokes equations (3) is then given by: find $\left(\boldsymbol{u}_{N}, p_{N}\right) \in V_{N}^{\boldsymbol{u}}\left(\Omega_{\boldsymbol{\mu}}\right) \times V_{N}^{p}\left(\Omega_{\boldsymbol{\mu}}\right)$, such that

$$
\begin{aligned}
& a\left(\boldsymbol{u}_{N}, \boldsymbol{v} ; \boldsymbol{\mu}\right)+c\left(\boldsymbol{u}_{N}, \boldsymbol{u}_{N}, \boldsymbol{v} ; \boldsymbol{\mu}\right)+b\left(\boldsymbol{v}, p_{N} ; \boldsymbol{\mu}\right) \\
& \quad-b\left(\boldsymbol{u}_{N}, q ; \boldsymbol{\mu}\right)+j_{p}\left(p_{N}, q\right)=l(\boldsymbol{v} ; \boldsymbol{\mu}) \quad \forall(\boldsymbol{v}, q) \in V_{N}^{\boldsymbol{u}}\left(\Omega_{\boldsymbol{\mu}}\right) \times V_{N}^{p}\left(\Omega_{\boldsymbol{\mu}}\right) .
\end{aligned}
$$

We now prove that problem (16) is well-posed and that its complexity can be simplified.

Theorem 1 Assume that $\left\{p^{(i)}\right\}_{i=1}^{N}$ are $\hat{j}_{p}$-linear independent. For a given parameter $\boldsymbol{\mu}$, problem (16) is then equivalent to: find $\boldsymbol{u}_{N}$ in $V_{N}^{\boldsymbol{u}}\left(\Omega_{\boldsymbol{\mu}}\right)$, say $\boldsymbol{u}_{N}=\sum_{i=1}^{N} \alpha_{i} \Psi_{\mu}^{-1}\left(\hat{\boldsymbol{u}}^{(i)}\right)$, such that

$$
a\left(\boldsymbol{u}_{N}, \boldsymbol{v}_{N} ; \boldsymbol{\mu}\right)+c\left(\boldsymbol{u}_{N}, \boldsymbol{u}_{N}, \boldsymbol{v}_{N} ; \boldsymbol{\mu}\right)+d\left(\boldsymbol{u}_{N}, \boldsymbol{v}_{N} ; \boldsymbol{\mu}\right)=l\left(\boldsymbol{v}_{N} ; \boldsymbol{\mu}\right) \quad \forall \boldsymbol{v}_{N} \in V_{N}^{\boldsymbol{u}}\left(\Omega_{\boldsymbol{\mu}}\right),
$$

where the bi-linear operator $d(\cdot, \cdot ; \boldsymbol{\mu}): V_{N}^{u}\left(\Omega_{\mu}\right) \times V_{N}^{u}\left(\Omega_{\mu}\right) \rightarrow \mathbb{R}$ is defined as

$$
d\left(\boldsymbol{u}_{N}, \boldsymbol{v}_{N} ; \boldsymbol{\mu}\right)=\sum_{i=1}^{N} \alpha_{i} b\left(\boldsymbol{v}_{N}, \hat{p}^{(i)} \circ \Phi_{\mu}^{-1} ; \boldsymbol{\mu}\right) .
$$

The pressure $p_{N}$ can then be computed with the coefficients of $\boldsymbol{u}_{N}$ as

$$
p_{N}=\sum_{i=1}^{N} \alpha_{i} \hat{p}^{(i)} \circ \Phi_{\mu}^{-1}
$$

We note that problem (17) is also non-linear, and we find the reduced basis solution by the same pseudo time-advancing scheme presented for the finite element problem (5). Since the dimension of the reduced basis problem is much smaller than the finite element problem, $N \ll \mathcal{N}$, we use a direct solver at each time-step. The time-advancing is then stopped when the difference in the reduced basis coefficients, $\left\{\alpha_{i}\right\}_{i=1}^{N}$, from one time-step to the next is below a certain limit, measured in the $l^{2}$-norm.

Assumption (15) requires a check in the construction of the basis-functions, e.g. by a supplementary Gram-Schmidt procedure.

Proof Let $\left(\boldsymbol{u}_{N}, p_{N}\right)$ be a solution of problem (16) and take $\boldsymbol{v}=0$. Then, for all $q_{N} \in$ $V_{N}^{p}\left(\Omega_{\mu}\right)$,

$$
\begin{aligned}
j_{p}\left(p_{N}, q_{N}\right) & =b\left(\boldsymbol{u}_{N}, q_{N} ; \boldsymbol{\mu}\right) \stackrel{(9)}{=} \hat{b}\left(\Psi_{\mu}^{-1}\left(\boldsymbol{u}_{N}\right), q_{N} \circ \Phi_{\boldsymbol{\mu}}\right) \\
& =\sum_{i} \alpha_{i} \hat{b}\left(\Psi_{\mu}^{-1}\left(\boldsymbol{u}^{(i)}\right), q_{N} \circ \Phi_{\mu}\right)=\sum_{i} \alpha_{i} \hat{b}\left(\left(\hat{\boldsymbol{u}}^{(i)}\right), q_{N} \circ \Phi_{\boldsymbol{\mu}}\right) \\
& \stackrel{(12)}{=} \hat{j}_{p}\left(\sum_{i=1}^{N} \alpha_{i} \hat{p}^{(i)}, q_{N} \circ \Phi_{\mu}\right)
\end{aligned}
$$


thanks to the linearity of the operator $\Psi_{\mu}$. From (15) we know that the only function in $V_{N}^{p}\left(\Omega_{\mu}\right)$ with this property is given by (19). Let now $q=0$ and $\boldsymbol{v} \neq 0, \boldsymbol{v} \in V_{N}^{u}\left(\Omega_{\mu}\right)$. Because of (19) and the definition (18), statement (17) holds.

If $\boldsymbol{u}_{N}$ is defined as in (17) and $p_{N}$ as in (19), then with similar arguments we have that $\left(\boldsymbol{u}_{N}, p_{N}\right)$ is a solution of problem (16).

As we see from this proof, when solving problem (17), the discretization of the Piola transformation affects only the pressure.

Corollary 1 Assume (15); for a given parameter $\boldsymbol{\mu}$, problem (17) is well-posed; as a consequence, also problem (16).

Proof Problem (17) is continuous because it is finite dimensional. Moreover, the continuity constant is bounded by the continuity constant of the stabilized finite element problem [3], thus independent of $N$.

To prove the coercivity, first note that when $\boldsymbol{u}_{N}$ is a solution of (17) and $p_{N}$ is defined by $(19), d\left(\boldsymbol{u}_{N}, \boldsymbol{u}_{N} ; \boldsymbol{\mu}\right)=b\left(\boldsymbol{u}_{N}, p_{N} ; \boldsymbol{\mu}\right)=j_{p}\left(p_{N}, p_{N}\right)>0$ (strict inequality follows from the property (15)). Then, from Lemma 3.3 in [3] we have that

$$
\begin{gathered}
a\left(\boldsymbol{u}_{N}, \boldsymbol{u}_{N} ; \boldsymbol{\mu}\right)+c\left(\boldsymbol{u}_{N}, \boldsymbol{u}, \boldsymbol{u}_{N} ; \boldsymbol{\mu}\right)+d\left(\boldsymbol{u}_{N}, \boldsymbol{u}_{N} ; \boldsymbol{\mu}\right) \\
\quad>a\left(\boldsymbol{u}_{N}, \boldsymbol{u}_{N} ; \boldsymbol{\mu}\right)+c\left(\boldsymbol{u}_{N}, \boldsymbol{u}, \boldsymbol{u}_{N} ; \boldsymbol{\mu}\right) \geq C\left\|\boldsymbol{u}_{N}\right\|^{2},
\end{gathered}
$$

for a mesh- and problem-dependent but $N$-independent norm $\|\cdot\| \|$.

\subsection{Remarks on the Computational Strategy}

The reduced basis method is decoupled in an offline and an online stage. Optimally, all computations with respect to the underlying finite element basis should be done in the offline stage. If the problem specific operators can be separated into a sum of products between parameter dependent functions and operators acting only on the basis-functions, e.g.

$$
a(\boldsymbol{u}, \boldsymbol{v} ; \boldsymbol{\mu})=\sum_{q=1}^{Q} g^{q}(\boldsymbol{\mu}) a^{q}(\boldsymbol{u}, \boldsymbol{v}),
$$

we have affine parameter dependence. It is then clear that all operator evaluations with respect to the basis-functions can be done independently of the given parameter, in the offline stage. In the online stage the contributions to the system matrix are then assembled from these precomputed values.

For problems where some derived quantity of the solution is desired, and not the solution itself, it is shown in [15] that for affine parameter dependence, the reduced basis approximation of this output of interest, together with rigorous error bounds, can be found in the online stage independent of the finite element degrees-of-freedom. In the case of non-affine parameter dependence, like usually with parametrized geometries, empirical interpolation may be employed to achieve the same independence; see [1].

Since we are interested in a proof-of-concept only, we have only implemented the bare essentials of the reduced basis element method. This means that we omit parts of the offline computations, like the non-affine decomposition and the necessary ingredients of the empirical interpolation used to compute the operator-evaluation of the basis-functions in the 
offline stage. Instead we compute the operator-evaluation of the basis-functions in the online stage. This slows down the online computation of the reduced basis approximation, but the approximation results are the same. For a detailed presentation of the offline-online decoupling of the reduced basis method for the Navier-Stokes problem when the geometry is used as a parameter, we refer the reader to $[6,10,18]$.

\section{Numerical Experiment}

To demonstrate the reduced basis method with stabilization, we construct an example where we seek the flow through a pipe which is found by contracting and bending the reference pipe. The deformation of the domain depends on two distinct parameters: the amplitude, $\mu_{1}$, of the contraction in the middle of the pipe, and the curvature, $\mu_{2}$, of the bent pipe. The deformed domain $\Omega_{\mu}$ in Fig. 2 is mapped starting from the straight reference pipe $\widehat{\Omega}$ of length $L=5$ and radius $r_{0}=0.5$ (Fig. 1) in two steps. First the radius, $r$, of the cross-section of the pipe is changed along the length, $l$, of the pipe according to

$$
r(l)=r_{0}+\frac{\mu_{1}}{2}\left(\cos \left(\frac{2 \pi l}{L}\right)-1\right) .
$$

Obviously, we must have $\mu_{1}<r_{0}$. Next the pipe is bent in a plane that contains the center axis of the pipe. In a Cartesian coordinate system, we let the $z$ axis be aligned with the center axis of the reference pipe, and assume that one end of the pipe is in the plane $z=0$. The center axis is rotated around the point $\left(1 / \mu_{2}, 0,0\right)$, and each plane perpendicular to the axis follows a rigid body motion. For a given point $\left(x_{0}, y_{0}, z_{0}\right)$, the new coordinates are given by

$$
\begin{aligned}
& x=\frac{1}{\mu_{2}}\left(1-\cos \left(\mu_{2} z_{0}\right)\right)+x_{0} \cos \left(\mu_{2} z_{0}\right), \\
& y=y_{0}, \\
& z=\frac{1}{\mu_{2}} \sin \left(\mu_{2} z_{0}\right)-x_{0} \sin \left(\mu_{2} z_{0}\right),
\end{aligned}
$$

Fig. 2 Example of a deformed pipe subject to both contraction and bending. In this case $\boldsymbol{\mu}=(0.25,0.25)$

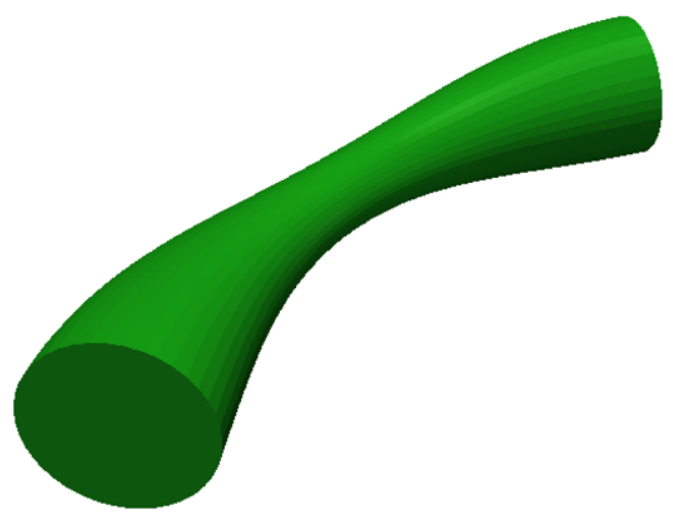


Table 1 The ordering of the parameters used to compute the basis-functions

\begin{tabular}{llllllll}
\hline$i$ & $\boldsymbol{\mu}=\left(\mu_{1}, \mu_{2}\right)$ & $i$ & $\boldsymbol{\mu}=\left(\mu_{1}, \mu_{2}\right)$ & $i$ & $\boldsymbol{\mu}=\left(\mu_{1}, \mu_{2}\right)$ & $i$ & $\boldsymbol{\mu}=\left(\mu_{1}, \mu_{2}\right)$ \\
\hline 1 & $(0.0,0.0)$ & 11 & $(0.3,0.8)$ & 21 & $(0.1,1.0)$ & 31 & $(0.25,0.6)$ \\
2 & $(0.4,1.0)$ & 12 & $(0.1,0.8)$ & 22 & $(0.3,0.0)$ & 32 & $(0.15,0.6)$ \\
3 & $(0.0,1.0)$ & 13 & $(0.3,0.3)$ & 23 & $(0.3,1.0)$ & 33 & $(0.25,0.4)$ \\
4 & $(0.4,0.0)$ & 14 & $(0.0,0.2)$ & 24 & $(0.1,0.5)$ & 34 & $(0.05,0.4)$ \\
5 & $(0.2,0.5)$ & 15 & $(0.4,0.7)$ & 25 & $(0.3,0.5)$ & 35 & $(0.35,0.6)$ \\
6 & $(0.0,0.5)$ & 16 & $(0.0,0.7)$ & 26 & $(0.05,0.1)$ & 36 & $(0.25,0.1)$ \\
7 & $(0.4,0.5)$ & 17 & $(0.4,0.2)$ & 27 & $(0.35,0.9)$ & 37 & $(0.15,0.9)$ \\
8 & $(0.2,0.0)$ & 18 & $(0.2,0.7)$ & 28 & $(0.05,0.9)$ & 38 & $(0.35,0.4)$ \\
9 & $(0.2,1.0)$ & 19 & $(0.2,0.2)$ & 29 & $(0.35,0.1)$ & & \\
10 & $(0.1,0.3)$ & 20 & $(0.1,0.0)$ & 30 & $(0.15,0.4)$ & & \\
\hline
\end{tabular}

where $z_{0}$ stands for the arc-length of the curved center axis; for $\left|\mu_{2}\right|<10^{-2}$ we replace (21) by the second order approximation

$$
\begin{aligned}
& x=\frac{\mu_{2} z_{0}^{2}}{2}+x_{0}\left(1-\frac{\mu_{2}^{2} z_{0}^{2}}{2}\right), \\
& y=y_{0}, \\
& z=z_{0}-\mu_{2} z_{0} x_{0} .
\end{aligned}
$$

Since the planes perpendicular to the axis are rotated, we must have $\mu_{2}<\frac{1}{r_{0}}$ to avoid intersection of the planes. (This restriction can locally be replaced by $\mu_{2}<\frac{1}{r(l)}$, but we consider constant bending along the entire pipe and from (20) we have $r_{0}>r(l)$.) In addition, to keep the inflow boundary from overlapping the outflow boundary, we must have $\mu_{2}<\frac{2 \pi}{L}$.

Note that in order to compute the Jacobian needed in the Piola transformation, we can either use the analytic description of the deformation presented in (20) and (21), or we can use the finite element basis more directly. We choose the latter approach, which also allows for computing the Jacobian when the analytic description of the deformation is not known.

Based on the deformation parameters $\mu_{1} \in[0,0.4]$ and $\mu_{2} \in[0,1.0]$ introduced above, we compute 38 velocity-pressure basis-functions by choosing combinations of the parameters in an ad-hoc optimal sense, in order to span large parts of the parameter space. From experience with other reduced-basis procedures, we try to resemble the choices of parameter combinations yielded by the Greedy-algorithm, without actually implementing it. Our choice of parameter combinations are given in an ordered fashion in Table 1, and the distribution in parameter space can be seen in Fig. 3 (black circles).

In the offline stage, the basis-functions are computed and orthonormalized. In the online stage, for new choices of parameters, we test the convergence of the error between the reduced basis approximation and the corresponding finite element solution of the steady Navier-Stokes problem with respect to the number of basis-functions used to compute the reduced basis approximation. The choice of parameters considered for the test are the red stars in Fig. 3; the intention is to place them far away from those used to build the reduced basis. 
Fig. 3 The distribution of parameters used to generate the basis-functions (in black circles), and the parameters used for the test cases $\boldsymbol{\mu}=(0.15,0.75)$, $(0.31,0.18),(0.32,0.68)$, $(0.1,0.16)$ (red stars). Here the values of $\mu_{1} \in[0,0.4]$ are given in the vertical direction, and the values of $\mu_{2} \in[0,1.0]$ in the horizontal direction

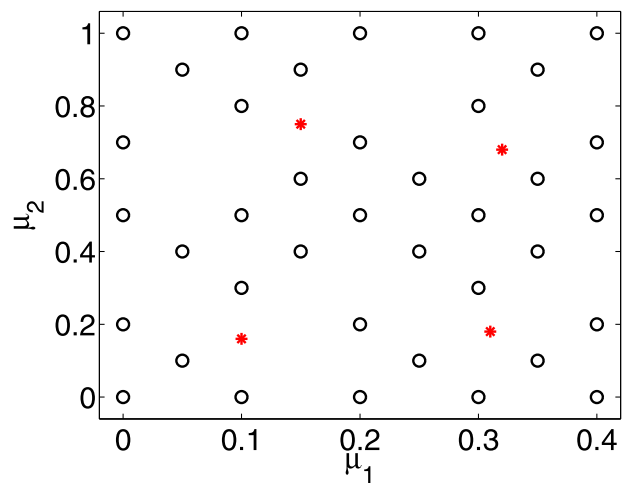

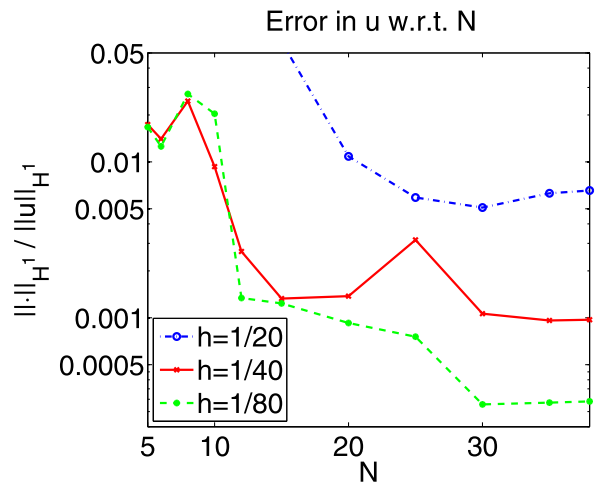

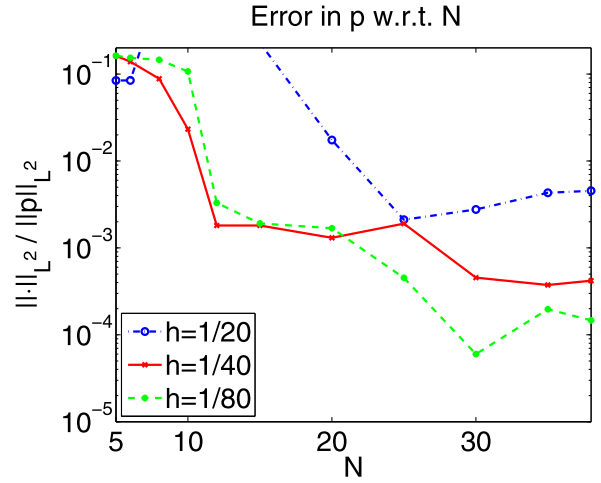

Fig. 4 Error behavior with increasing reduced basis dimension and comparison with different reference meshes

In Fig. 4 we present the maximal relative errors over the four chosen values of the parameter when using the proposed split representation of velocity and pressure (17)-(19). The error in the velocity is measured with respect to the $H^{1}$-norm and the error in the pressure is measured with respect to the $L^{2}$-norm. Since we do not use a Greedy-algorithm for the generation of the basis-functions, the "usual" exponential convergence is absent. We have performed the same error analysis on thirty more parameters equally distributed in the parameter domain. The convergence graphs are essentially the same, although for many of these thirty parameters the errors are smaller (one order of magnitude), since they are near to the location of the parameters chosen to generate the basis-functions.

Both the velocity error and the pressure error depend on the mesh used in the underlying finite element approximation. With a finer mesh we get also better reduced basis approximation. This is largely due to the discretization of the Piola mapping, and hence on the resolution of the (stabilized) divergence equation. We also remark that the finite element resolution induces a plateau that can be lowered with finer meshes (cf. in particular the velocity error for $N>30$ ).

The definition of the stabilizing term (4) plays an important role in the approximation; we tried in fact to compute it on the deformed domain instead of on the reference domain and the results were one order of magnitude worse. In fact, the developments in Sect. 3.2 show 


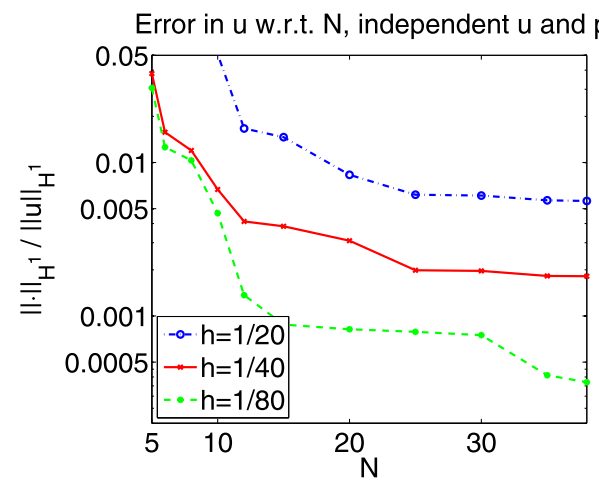

Error in $\mathrm{p}$ w.r.t. $\mathrm{N}$, independent $\mathrm{u}$ and $\mathrm{p}$

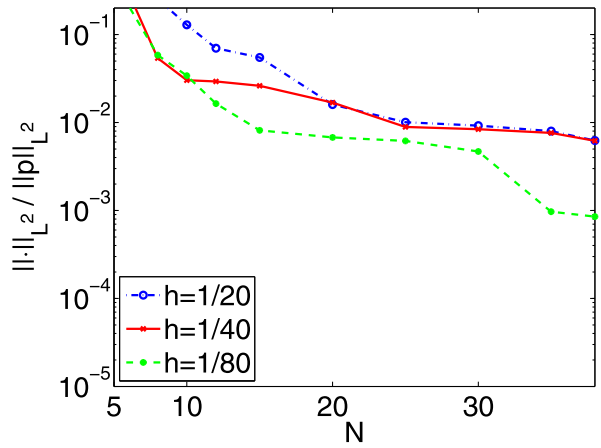

Fig. 5 Similarly to Fig. 4 we plot the error w.r.t. $N$. Here instead of considering the proposed splitting scheme, we consider a monolithic system (the scales w.r.t. Fig. 4 are the same)
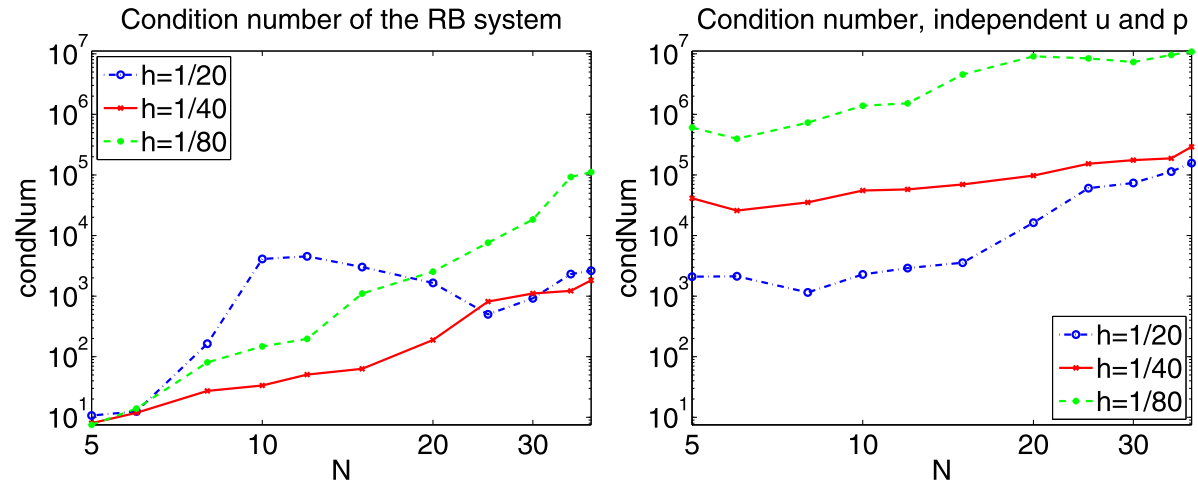

Fig. 6 Behavior of the condition number with increasing reduced basis dimension and comparison with different reference meshes. On the left we consider the proposed splitting scheme, on the right, a monolithic system

that there can be a deteriorations of the approximation due to the discretization of the Piola transformation. Using (4) the effects are attenuated thanks to simplifications at the algebraic level.

We ran the same simulations by solving problem (16). The first note on the results presented in Fig. 5 is that the convergence with respect to a growing number of basis functions $N$ is monotone, while this is not always the case with the split representation. However, the precision obtained for the pressure is one order of magnitude worse, although that for the velocity is only slightly worse.

We also compared (Fig. 6) the condition number of the reduced systems. Except for $h=1 / 20$, the condition number of the simplified problem (17) is two orders of magnitude smaller than that of (16). This is due essentially to two factors: firstly, when dealing with the split procedure, the unknowns are only the velocity components and is not a saddle point problem, secondly, the full system includes the stabilizing terms, which introduces artificial eigenvalues that depends on $h$. 


\section{Conclusions}

We have presented the reduced basis method applied to the incompressible Navier-Stokes equations, together with a procedure for stabilizing the resulting discrete problem. As opposed to earlier works for applying the reduced basis method to the Navier-Stokes equations, this gives us two benefits. First, we avoid having to enrich the reduced basis velocity space in order to satisfy the inf-sup condition related to the equations. Second, due to the relation between the stabilization term and the divergence operator, we are able to choose a reduced basis solution where the velocity is found by the solution of a small and well conditioned system, and then the pressure is recovered by simple linear combination. The resulting reduced basis system is thus a third of the size when compared to the reduced basis method without the stabilizing procedure and half the size when compared to a monolithic treatment of velocity and pressure.

A crucial point in the reduced basis procedure is in the accuracy of the Piola transformation. Analytically, the computation of the divergence of a velocity field on the reference domain is the same as computing the divergence of the Piola transformed field on a deformed domain. In the discrete setting this equality no longer holds, and we have to take this into account in our approximation.

Acknowledgement This work has been supported by the Research Council of Norway through a Centre of Excellence grant to the Center for Biomedical Computing. The support is gratefully acknowledged.

\section{References}

1. Barrault, M., Maday, Y., Nguyen, N.C., Patera, A.T.: An 'empirical interpolation' method: Application to efficient reduced-basis discretization of partial differential equations. C. R. Acad. Sci. Paris, Ser. I 339, 667-672 (2004)

2. Brezzi, F., Fortin, M.: Mixed and Hybrid Finite Element Methods. Springer, Berlin (1991)

3. Burman, E., Fernández, M.A., Hansbo, P.: Continuous interior penalty finite element method for Oseen's equations. SIAM J. Numer. Anal. 44(3), 1248-1274 (2006)

4. Chen, Y., Hesthaven, J.S., Maday, Y.: A seamless reduced basis element method for 2d maxwell's problem: An introduction. In: Barth, T.J., Griebel, M., Keyes, D.E., Nieminen, R.M., Roose, D., Schlick, T., Hesthaven, J.S., Rønquist, E.M. (eds.) Spectral and High Order Methods for Partial Differential Equations. Lecture Notes in Computational Science and Engineering, vol. 76, pp. 141-152. Springer, Berlin-Heidelberg (2011)

5. Deparis, S.: Reduced basis error bound computation of parameter-dependent Navier-Stokes equations by the natural norm approach. SIAM J. Numer. Anal. 46(4), 2039-2067 (2008)

6. Deparis, S., Rozza, G.: Reduced basis method for multi-parameter dependent steady Navier-Stokes equations: applications to natural convection in a cavity. J. Comput. Phys. 228(12), 4359-4378 (2009). EPFLIACS report 12.2008

7. LifeV. http://www.lifev.org

8. Løvgren, A.E., Maday, Y., Rønquist, E.M.: A reduced basis element method for the steady Stokes problem. Modél. Math. Anal. Numér. 40(3), 529-552 (2006)

9. Løvgren, A.E., Maday, Y., Rønquist, E.M.: The reduced basis element method for fluid flows. In: Calgaro, C., Coulombel, J.-F., Goudon, T. (eds.) Analysis and Simulation of Fluid Dynamics. Advances in Mathematical Fluid Mechanics, pp. 129-154. Birkhäuser, Basel (2007)

10. Løvgren, A.E., Maday, Y., Rønquist, E.M.: The reduced basis element method: Offline-online decomposition in the nonconforming, nonaffine case. In: Barth, T.J., Griebel, M., Keyes, D.E., Nieminen, R.M., Roose, D., Schlick, T., Hesthaven, J.S., Rønquist, E.M. (eds.) Spectral and High Order Methods for Partial Differential Equations. Lecture Notes in Computational Science and Engineering, vol. 76, pp. 247254. Springer, Berlin-Heidelberg (2011)

11. Maday, Y., Rønquist, E.M.: A reduced-basis element method. J. Sci. Comput. 17, 447-459 (2002)

12. Maday, Y., Rønquist, E.M.: The reduced-basis element method: Application to a thermal fin problem. SIAM J. Sci. Comput. 26(1), 240-258 (2004) 
13. Noor, A.K., Peters, J.M.: Reduced basis technique for nonlinear analysis of structures. AIAA J. 18(4), 455-462 (1980)

14. Peterson, J.S.: The reduced basis method for incompressible viscous flow calculations. SIAM J. Sci. Stat. Comput. 10(4), 777-786 (1989)

15. Prud'homme, C., Rovas, D.V., Veroy, K., Machiels, L., Maday, Y., Patera, A.T., Turinici, G.: Reliable real-time solution of parametrized partial differential equations: Reduced basis output bound methods. J. Fluids Eng. 124, 70-80 (2002)

16. Rozza, G.: Reduced-basis methods for elliptic equations in sub-domains with a posteriori error bounds and adaptivity. Appl. Numer. Math. 55, 403-424 (2005)

17. Rozza, G., Veroy, K.: On the stability of the reduced basis method for Stokes equations in parametrized domains. Comput. Methods Appl. Mech. Eng. 196(7), 1244-1260 (2007)

18. Rozza, G.: Reduced basis methods for Stokes equations in domains with non-affine parameter dependence. Comput. Vis. Sci. 12(1), 23-35 (2009)

19. Winkelmann, C.: Interior penalty finite element approximation of Navier-Stokes equations and application to free surface flows. Ph.D. Thesis, École Polytechnique Fédérale de Lausanne, December 2007 